\title{
Periodontal Status and Relation with Salivary Total Antioxidant Capacity after Green Tea Consumption in Smokers
}

\author{
${ }^{1}$ Sedigheh Bakhtiari, ${ }^{2}$ Somayyeh Azimi, ${ }^{3}$ Zahra Mansouri, ${ }^{4}$ Estie Kruger, ${ }^{5}$ Marc Tennant, ${ }^{6}$ Mahshid Namdari
}

\begin{abstract}
Aim: Smoking is a significant source of oxidative stress. Also, the disruption in equilibrium between free-oxygen radicals and antioxidants plays a pivotal role in the progression of periodontal inflammation. Green tea with antioxidant potential might have an effect on periodontal disease. This study evaluated the periodontal status in different groups of smokers compared with nonsmokers and investigates the association with salivary antioxidant levels after a period of green tea consumption.
\end{abstract}

Materials and methods: In this interventional study, 60 healthy males, including 20 light and 20 heavy cigarette smokers and 20 control nonsmokers, participated. Periodontal status was determined by the Community Periodontal Index of Treatment Needs (CPITN) at the beginning of the study. All participants were asked to consume a total of two cups of green tea (4 gm) per day. Total antioxidant capacity (TAC) of saliva was measured at baseline and after 21 days. Statistical evaluation was done by Statistical Package for the Social Sciences (SPSS) version 21 software.

Results: The Pearson correlation coefficient between CPITN and salivary TAC showed significant correlation in light and heavy smokers ( $p=0.001$ and $p=0.003$ respectively). Changes in salivary TAC from baseline to day 21 , after green tea consumption, in subjects with CPITN $\leq 18$ were $139( \pm 61.5)$, and $66.16( \pm 67.37)$ in subjects with CPITN $>18$. There was a significant interaction effect between time of TAC evaluation and patients' periodontal state $(p=0.009)$.

Conclusion: This study confirmed the association between periodontal status and smoking, and the association with salivary antioxidant capacity. A significant alteration in TAC of whole saliva in cases with clinical periodontal problems after green tea consumption was indicated.

\footnotetext{
${ }^{1,4}$ Associate Professor, ${ }^{2}$ Honorary Research Fellow, ${ }^{3,6}$ Assistant Professor, ${ }^{5}$ Professor

${ }^{1}$ Department of Oral Medicine, Shahid Behesti University of Medical Sciences, Tehran, Islamic Republic of Iran

2,4,5 Department of Human Sciences, University of Western Australia, Perth, Western Australia, Australia

${ }^{3}$ Department of Oral Medicine, Yasuj University of Medical Sciences, Yasuj, Islamic Republic of Iran

${ }^{6}$ Community Oral Health Department, Shahid Behesti University of Medical Sciences, Tehran, Islamic Republic of Iran

Corresponding Author: Somayyeh Azimi, Honorary Research Fellow, Department of Human Sciences, University of Western Australia, Perth, Western Australia, Australia, Phone: +61864883290 e-mail: somayyeh.azimi@uwa.edu.au
}

Clinical significance: Considering the safety and availability of green tea, it can be used as a preventive or supplementary treatment in periodontal problems, especially in smokers, after further investigation.

Keywords: Antioxidant capacity, Green tea, Periodontal, Saliva, Smokers.

How to cite this article: Bakhtiari S, Azimi S, Mansouri Z, Kruger E, Tennant M, Namdari M. Periodontal Status and Relation with Salivary Total Antioxidant Capacity after Green Tea Consumption in Smokers. Int J Experiment Dent Sci 2017;6(2):61-64.

Source of support: School of Dentistry, Shahid Beheshti University of Medical Sciences, Tehran, Islamic Republic of Iran.

Conflict of interest: None

\section{INTRODUCTION}

Periodontitis is an inflammatory disease caused by imbalance between the levels of bacterial pathogens and the host immune response to infection. Investigations have demonstrated that disruption in equilibrium between free-oxygen radicals and antioxidants [due to production of more reactive oxygen species (ROS) against microorganisms and consequent oxidative stress] plays a pivotal role in the progression of periodontal inflammation. ${ }^{1,2}$ On the contrary, smoking is a significant source of oxidative stress by increases in ROS production. ${ }^{3-5}$ The exact mechanism by which smoking exerts effects on the periodontium is unclear. ${ }^{3}$ It has been shown that smoking increases the levels of free radicals and lipid peroxidation in periodontal tissues and subsequently decreased antioxidant levels in blood, saliva, and gingival crevicular fluid (GCF) in patients with periodontitis and gingivitis who smoke. ${ }^{6}$

Antioxidants could scavenge the free radicals and maintain the balance between oxidants and antioxidants. Therefore, antioxidant therapy as an adjunctive treatment by reducing the oxidative stress in periodontal tissues has been advocated enhancing the effects of periodontal therapy. ${ }^{7}$

Green tea is a herbal product containing several polyphenolic components, classified as catechins, including epicatechin, epigallocatechin, epicatechin gallate, and epigallocatechin gallate, that have been shown to possess potent antioxidant property. ${ }^{1,7}$

In the field of periodontology, green tea showed an antibacterial effect against periodontopathic bacteria. 
Also, it has been shown that green tea polyphenols might completely inhibit the collagenase activity in the GCF in progressive periodontitis patients. Green tea may also prevent alveolar bone resorption, inhibit the catalytic activity of neutrophil elastase and Porphyromonas gingivalis collagenase, and dose dependently inhibit the secretion of interleukin (IL)-6, IL-8, and chemokine (C-C motif) ligand 5 (CCL-5). ${ }^{8}$ Priya et $\mathrm{al}^{9}$ and Sarin et al, ${ }^{10}$ both randomized clinical studies, concluded that green tea-containing mouthwash could be effective in reducing gingival inflammation.

Furthermore, an epidemiological study by Kushiyama et $\mathrm{al}^{11}$ concluded that there was a modest inverse association between the intake of green tea and periodontal disease. However, it is still unclear how consumption of green tea affects salivary oxidative stress in smokers. ${ }^{2}$

In a previous study, we indicated that green tea improves the salivary antioxidant status in both cigarette smokers (CS) and nonsmokers (NS), and we found a significant difference in salivary TAC alteration in CS compared with NS over the study period. ${ }^{12}$ Against this background, we hypothesize that daily consumption of green tea might have different effects on the periodontal status of different groups (categorized based on their smoking or nonsmoking status) and might contribute to decreased gingival oxidative stress. Therefore, the main objectives of this study were to compare the periodontal status of different groups of smokers and nonsmokers, determine the relation between TAC and periodontal status, and investigate the alteration in TAC after green tea consumption.

\section{MATERIALS AND METHODS}

\section{Ethics}

The study was conducted according to the principles of the "Declaration of Helsinki" and approved by the Ethics Committee of the Shahid Beheshti University of Medical Sciences. All participants signed an informed consent document prior to the study.

\section{Study Protocol}

This study is a following of our previous work that dealt with the effect of green tea on salivary antioxidant alteration in smokers. ${ }^{12}$ Considering the power and sample size calculation, we recruited 60 healthy male participants (from attendees to the oral medicine department of a dental school) for this interventional study, 20 in each group. Group I included 20 healthy male light CS; group II included 20 healthy male light CS, and the control group included 20 healthy male NS. Smokers were defined as those who have ever smoked 100 cigarettes over their lifetime and had smoked daily in the past 30 days $^{13}$; and light CS were defined as those who smoked less than 10 cigarettes/day, and heavy CS who smoked equal to or more than 10 cigarettes/day. ${ }^{3,13}$ Also, NS were defined as those who self-reported never having smoked. Also, CS and NS were matched in terms of age.

The CPITN was used to provide a detailed assessment of gum health in all participants. A graduated periodontal probe inserted between teeth and gums at a standard force (approximately 20-25 gm) was used to measure pocket depth by one trained dentist, as well as assessment of calculus accumulation on the subgingival surfaces of the teeth. These evaluations were made for each sextant of the dentition. If second molars were missing, the third molars were included. The scoring index for this procedure ranged from 0 to 4 [no disease, bleeding on probing (BOP), calculus without pocketing, shallow or deep periodontal pockets]. Final periodontal disease scores were calculated by taking the sum of the sextant scores and subtracting this figure from a maximum of 24. Therefore, in this study, a high CPITN score represents healthy gingivae and a low score indicates disease. ${ }^{14}$ Also, we divided participants into two groups, according to CPITN scores. A case definition for clinically significant periodontal disease was defined as CPITN $\leq 18$, based on previous studies. ${ }^{14}$ For elimination of confounding bias, patients who had received any surgical or nonsurgical periodontal therapy in the past 6 months and had a history of use of antibiotics, antimicrobial mouth rinse, anti-inflammatory agents, and any chemotherapeutics in the past 3 months or patients with $<20$ teeth were excluded from study. ${ }^{10}$

All participants were instructed to consume a total of two cups of green tea ( 2 gm of green tea in $150 \mathrm{~mL}$ of hot water for 3 minutes) per day, for a period of 3 weeks. Whole unstimulated saliva was collected, and TAC was measured and compared at baseline, and after 3 weeks of drinking green tea. The inclusion criteria, experimental protocol, saliva collection, and TAC assay were the same as our previously published study. ${ }^{12}$

\section{Statistical Analysis}

Results were expressed as mean \pm standard deviation. The differences between groups were assessed by ANOVA and sample t-tests. We assumed a p-value of $<0.05$ (95\% confidence level) to specify a significant difference. The SPSS 21 software was used for statistical analyzing.

\section{RESULTS}

In the present study, 60 healthy males participated in three groups. The periodontal status of each group in terms of CPITN is shown in Graph 1. Table 1 compares 


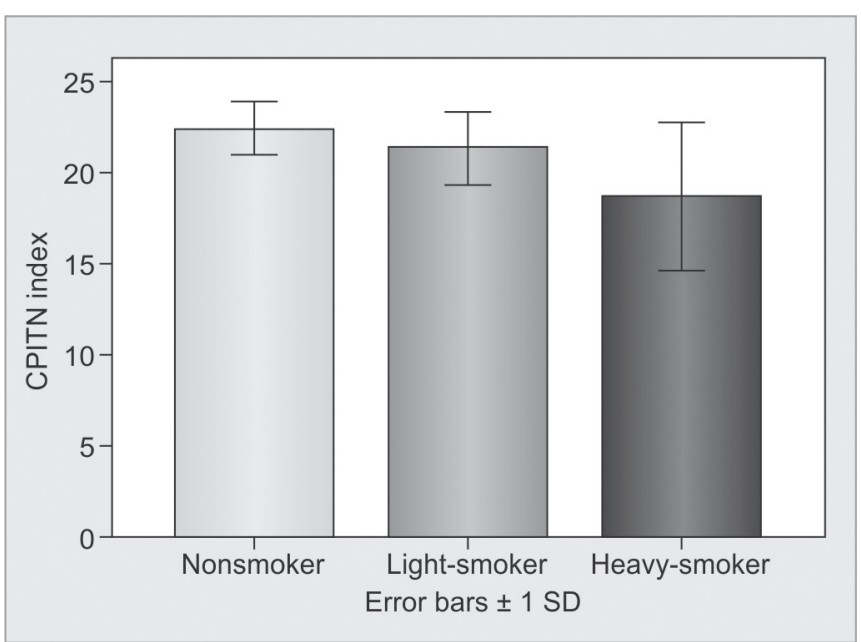

Graph 1: Mean of CPITN index in study groups

Table 1: Differences in CPITN between groups

\begin{tabular}{ll}
\hline $\begin{array}{l}\text { Compare means between each } \\
\text { of the two groups }\end{array}$ & $p$-value \\
\hline NS-light CS & 0.185 \\
NS-Heavy CS & 0.002 \\
Light CS-heavy CS & 0.032 \\
\hline
\end{tabular}

Table 2: Alteration in salivary TAC from baseline to day 21 in two groups considering periodontal disease

\begin{tabular}{ll}
\hline Periodontal group & Salivary TAC alteration \\
\hline CPITN $\leq 18$ & $139( \pm 61.5)$ \\
CPITN $>18$ & $66.16( \pm 67.37)$ \\
p-value & 0.009 \\
\hline
\end{tabular}

the difference in CPITN score between each of the two groups using a robust test of one-way ANOVA (Welch test) and Tamhane's post hoc test (Table 1). It indicated the significant difference in periodontal index between NS and heavy CS and between light CS and heavy CS. Also, the Pearson correlation coefficient between periodontal status index (CPTIN) and salivary TAC was $0.29(\mathrm{p}=0.21)$ in NS, $0.69(p=0.001)$ in light $C S$, and $0.64(p=0.003)$ in heavy CS groups respectively; that was significant in light and heavy CS. Table 2 indicates the alteration in salivary TAC from baseline to day 21 , with a case definition for clinically significant periodontal disease considered as a CPITN $\leq 18$ (Table 2). There was a significant interaction effect between time of TAC evaluation and patients' periodontal state $(\mathrm{p}=0.009)$. It means that the average change in TAC was not the same between healthy subjects and those with periodontal disease. Comparing TAC levels between healthy subjects and those with periodontal disease at baseline and day 21 separately showed significant differences between the two groups of patients using Bonferroni post hoc test $(\mathrm{p}<0.0001, \mathrm{p}<0.0001)$.

However, in the heavy CS group, changes in salivary TAC levels were not statistically significant between subjects with CPITN $>18$ and subjects with CPITN $\leq 18$ $(\mathrm{p}=0.729)$.

\section{DISCUSSION}

The current study indicated the difference in CPITN index and salivary TAC in NS $v$ s light and heavy CS after green tea consumption. The whole unstimulated saliva was used for measuring TAC. Stimulating salivary flow might have increased saliva volume, but would have disrupted the antioxidant concentration. Also, the mastication process that might be used for salivary stimulation may cause the expulsion of relatively high quantities of GCF from the periodontal pockets, artificially increasing the antioxidant capacity in saliva with plasma-derived antioxidants. ${ }^{14}$

The results of present study showed that heavy CS had worse periodontal status as indicated by lower CPITN scores. There is an abundance of literature supporting the relationship between smoking and periodontal diseases. ${ }^{14,15}$ The induction of oxidative stress by nicotine and the depletion of antioxidants subsequently might be one of the mechanisms for the tissue damage. ${ }^{14}$ The finding of this study demonstrated that subjects with the worse periodontal health status tended to have greater oxidative damage, as shown by the presence of lower salivary TAC in whole saliva. ${ }^{14}$ Agnihotri et $\mathrm{al}^{3}$ reported a progressive reduction in salivary superoxide dismutase (SOD) levels from healthy NS to light CS to heavy CS in subjects with chronic periodontitis. They argued that reduced smoking might improve antioxidant levels. Tonguç et $\mathrm{al}^{6}$ argued the impact of smoking status on antioxidant enzyme activity in subjects with chronic periodontitis and concluded that both periodontitis and smoking lead to significant changes in antioxidant enzyme activity in blood and gingival tissues. The combination of smoking and periodontitis resulted in significant alterations in SOD activities in periodontium.

The main finding of this study demonstrated that subjects with a lower CPITN index and worse periodontal health had a higher change in salivary TAC levels after a 3 -week green tea consumption. Kushiyama et a $1^{11}$ analyzed periodontal status of 940 Japanese men, and evaluated the association between the consumption of green tea, which was determined by a self-reported questionnaire for the number of cups per day, and periodontal parameters. The multivariate linear regression models showed every one cup per day increment in green tea intake was significantly associated with a decrease in the mean probing depth, clinical attachment loss, and a decrease in BOP. They concluded the almost inverse relationship between the green tea drinking and periodontal disease. 
To evaluate the role of green tea consumption as an adjunct to periodontal therapy for the management of mild-to-moderate chronic periodontitis and effect on the TAC on GCF and plasma, Chopra et $\mathrm{al}^{16}$ ran a clinical trial on 120 subjects and demonstrated a significant improvement in all clinical parameters along with an 8 -fold greater antioxidant capacity in GCF in the green tea-intake group than nonconsumers.

In the present study, considering the taste as well as the color of green tea, it was practically impossible to have a placebo group. Also, we could not recruit female smokers because of low prevalence and cultural issues in Islamic Republic of Iran. Smoking status was recorded based on self-reporting by participants and there was a lack of chemical validation of the smoking status; however, most of the previous studies used self-reporting status. In this study, we measured periodontal status only at baseline. Considering the length of study and the fact that participants did not get any mechanical periodontal therapy in the study period, it seems that measuring CPITN again at the end of study would not be a good evaluation.

\section{CONCLUSION}

With the limitation of the study, we can conclude that green tea intake increases the TAC of whole saliva in adults with significant periodontal problems due to smoking, and might be useful as a preventive or adjunct periodontal treatment in this group of people.

\section{CLINICAL SIGNIFICANCE}

Based on results, considering the safety and availability of green tea, it can be used as a preventive or supplementary treatment in periodontal problems, especially in smokers, after further investigation.

\section{ACKNOWLEDGMENT}

This project has been supported by School of Dentistry, Shahid Beheshti University of Medical Sciences, Tehran, Islamic Republic of Iran. Dr Azimi acknowledges the Australian Government support through an Australian Government Research Training Program Scholarship from the University of Western Australia.

\section{REFERENCES}

1. Maruyama T, Tomofuji T, Endo $Y$, Irie K, Azuma T, Ekuni D, Tamaki N, Yamamoto T, Morita M. Supplementation of green tea catechins in dentifrices suppresses gingival oxidative stress and periodontal inflammation. Arch Oral Biol 2011 Jan;56(1):48-53.
2. Hrishi TS, Kundapur PP, Naha A, Thomas BS, Kamath S, Bhat GS. Effect of adjunctive use of green tea dentifrice in periodontitis patients - a randomized controlled pilot study. Int J Dent Hyg 2016 Aug;14(3):178-183.

3. Agnihotri R, Pandurang P, Kamath SU, Goyal R, Ballal S, Shanbhogue AY, Kamath U, Bhat GS, Bhat KM. Association of cigarette smoking with superoxide dismutase enzyme levels in subjects with chronic periodontitis. J Periodontol 2009 Apr;80(4):657-662.

4. Sedighe B, Maryam B, Fahimeh A, Somayyeh A, Bigom TJ. Effect of vitamin $C$ on salivary superoxide dismutase activity in smokers. Afr J Biotech 2011 Jul;10(37):7267-7270.

5. Bakhtiari S, Azimi S, Mehdipour M, Amini S, Elmi Z, Namazi Z. Effect of cigarette smoke on salivary total antioxidant capacity. J Dent Res Dent Clin Dent Prospects 2015 Fall;9(4):281-284.

6. Tonguç MÖ, Öztürk Ö, Sütçü R, Ceyhan BM, Kilinç G, Sönmez Y, Yetkin Ay Z, Sahin U, Baltacıŏlu E, Kırzıŏlu FY. The impact of smoking status on antioxidant enzyme activity and malondialdehyde levels in chronic periodontitis. J Periodontol 2011 Sep;82(9):1320-1328.

7. Nugala B, Namasi A, Emmadi P, Krishna PM. Role of green tea as an antioxidant in periodontal disease: the Asian paradox. J Indian Soc Periodontol 2012 Jul-Sep;16(3):313-316.

8. Rattanasuwan K, Rassameemasmaung S, Sangalungkarn V, Komoltri C. Clinical effect of locally delivered gel containing green tea extract as an adjunct to non-surgical periodontal treatment. Odontology 2016 Jan;104(1):89-97.

9. Priya BM, Anitha V, Shanmugam M, Ashwath B, Sylva SD, Vigneshwari SK. Efficacy of chlorhexidine and green tea mouthwashes in the management of dental plaque-induced gingivitis: a comparative clinical study. Contemp Clin Dent 2015 Oct-Dec;6(4):505-509.

10. Sarin S, Marya C, Nagpal R, Oberoi SS, Rekhi A. Preliminary clinical evidence of the antiplaque, antigingivitis efficacy of a mouthwash containing $2 \%$ green tea-A randomised clinical trial. Oral Health Prev Dent 2015 Jan;13(3):197-203.

11. Kushiyama M, Shimazaki Y, Murakami M, Yamashita Y. Relationship between intake of green tea and periodontal disease. J Periodontol 2009 Mar;80(3):372-377.

12. Azimi S, Mansouri Z, Bakhtiari S, Tennant M, Kruger E, Rajabibazl M, Daraei A. Does green tea consumption improve the salivary antioxidant status of smokers? Arch Oral Biol 2017 Jun;78:1-5.

13. Boulos DN, Loffredo CA, El Setouhy M, Abdel-Aziz F, Israel E, Mohamed MK. Nondaily, light daily, and moderate-to-heavy cigarette smokers in a rural area of Egypt: a population-based survey. NicoTob Res 2009 Feb;11(2):134-138.

14. Sculley DV, Langley-Evans SC. Periodontal disease is associated with lower antioxidant capacity in whole saliva and evidence of increased protein oxidation. Clin Sci (Lond) 2003 Aug;105(2):167-172.

15. Calsina G, Ramón JM, Echeverría JJ. Effects of smoking on periodontal tissues. J Clin Periodontol 2002 Aug;29(8):771-776.

16. Chopra A, Thomas BS, Sivaraman K, Prasad HK, Kamath SU. Green tea intake as an adjunct to mechanical periodontal therapy for the management of mild to moderate chronic periodontitis: a randomized controlled clinical trial. Oral Health Prev Dent 2016;14(4):293-303. 